\title{
Pseudopregnancy and aseasonal breeding in dairy goats: genetic basis of fertility and impact on lifetime productivity
}

\author{
S. Desire ${ }^{1}$, S. Mucha ${ }^{1 \dagger}$, M. Coffey ${ }^{1}$, R. Mrode ${ }^{1,2}$, J. Broadbent $^{3}$ and J. Conington ${ }^{1}$ \\ ${ }^{1}$ Animal and Veterinary Sciences, SRUC (Scotland's Rural College), West Mains Road, Edinburgh EH9 3J G, Scotland, UK; ${ }^{2}$ Animal Biosciences, International Livestock \\ Institute, 30709 Naivasha Rd, Nairobi, Kenya; ${ }^{3}$ Yorkshire Dairy Goats, St Helen's Farm, Seaton Ross, York YO42 4NP, UK
}

(Received 20 December 2016; Accepted 5 September 2017; First published online 1 December 2017)

\begin{abstract}
Until recently, the main selection focus in UK dairy goats has been on milk yield. To develop a selection index suitably weighted for a variety of traits, it is important to understand the genetic relationships between production, health and fertility traits. This study focussed on three aspects of reproduction that are of interest to goat breeders. (1) Out of season (OOS) kidding ability: goats are highly seasonal breeders so achieving consistent, year-round dairy production presents a challenge. It may be possible to select for extended or shifted breeding cycles, however, there are no published studies on the genetic basis of seasonal kidding ability, and a genetic correlation with milk production in dairy goats; (2) age at first kidding (AFK): a reduced AFK offers the opportunity for more rapid genetic improvement, as well as reducing the amount of time and resources required to raise the animals to producing age; (3) pseudopregnancy (PPG): as it is difficult to diagnose pregnancy within 30 days of mating, high herd levels of PPG could add a significant delay in breeding replacement animals, or commencing a new lactation. Using records from 9546 goats, the objective of this study was to investigate the genetic relationships between the reproductive traits described above, and the production traits 520-day milk yield (MY520), lifetime milk yield (MYLife) and lifetime number of days in milk (DIMLife). The 'out of season' phenotype was defined as week of kidding relative to the 4 weeks of the year where the highest average number of births occur. Incidences of PPG that occurred during the first lactation were used as cases, while goats with none were assigned as controls. Relevant fixed and random effects were fitted in the models. In line with other reproduction traits, heritability estimates were low ranging from 0.08 to 0.11 . A negative genetic correlation was found between AFK and MY520 $(-0.22 \pm 0.10)$, whereas a positive genetic correlation was found between PPG and DIMLife $(0.58 \pm 0.11)$. Pseudopregnancy and OOS were positively genetically correlated $(0.36 \pm 0.15)$. All other genetic correlations were very low. The results of this study indicate that selection for the reproductive traits analysed is feasible, without adversely affecting MYLife.
\end{abstract}

Keywords: dairy goats, genetics, fertility, pseudopregnancy, out of season kidding

\section{Implications}

Seasonal reproduction presents a challenge when supplying year-round goat milk, and selection for aseasonal breeding ability may help alleviate this problem. However, measuring out of season breeding ability is difficult, as mating records are not routinely collected in naturally bred herds. We developed a method of measuring out of season (OOS) kidding ability using routinely collected birth records. This study demonstrated that selection for this trait may be possible, without impairing milk production. We also showed that pseudopregnancy (PPG), which is common in goats, is genetically associated with longer productive lifespan, but not with milk yield.

${ }^{\dagger}$ Present address: Poznan University of Life Sciences, 33 Wolynska, 60-637 Poznan, Poland. E-mail: sebastian.mucha@sruc.ac.uk

\section{Introduction}

Year-round dairy production is important when supplying fresh milk, but can be difficult to achieve in species with narrow seasonal breeding patterns, such as goats. The breeding season for goats in temperate regions typically starts in September and ends in February (northern hemisphere). Peak kidding season (PKS) occurs in early spring, whereas late autumn births are uncommon. Producers have historically attempted to mitigate this problem by extending lactation length, however, milk yields decrease over the course of the lactation period, affecting herd milk output, and high genetic merit females with extended lactations have fewer opportunities to contribute high-merit herd replacements. Selection for year-round breeding would alleviate this problem, however, there are many challenges associated with accurately recording this trait on large, naturally mated dairy herds (Hanocq et al., 1999). 
Pseudopregnancy is relatively common in dairy goats and is typically diagnosed via ultrasound, performed more than 30 days after insemination or breeding. Although it is clear that environmental factors largely influence $P P G$, there is some evidence that this condition is partially under genetic control (Hesselink, 1993). Age at first kidding (AFK) is an economically important trait to milk producers, due to the costs associated with feeding, housing and veterinary care for unproductive animals. Selection for reduced AFK in dairy goats would allow for more rapid genetic improvement, as well as reducing management costs.

Placing too much emphasis on production efficiency, whilst neglecting other traits, may result in unintended and unwanted consequences on the health and productivity of animals, as shown in other species (Rauw et al., 1998). This study therefore had three main objectives: (1) investigate the feasibility of measuring OOS kidding using routinely collected data; (2) estimate genetic parameters for 'out of season' kidding, PPG and AFK in UK dairy goats, as a first step towards developing genetic evaluations for these traits; (3) calculate phenotypic and genetic correlations between these traits and three production traits - 520-day milk yield (MY520) within the first lactation, lifetime milk yield (MYLife) and total lifetime days in milk (DIMLife) (as a proxy measure for longevity); in order to determine the relationship of these traits with production.

\section{Material and methods}

\section{Animals and management}

Records from continually indoor housed dairy goats located on two farm sites between $53^{\circ}$ and $54^{\circ}$ latitudes north were used in this study. The goats were a synthetic population of crossbred dairy goats of three original breeds (Alpine, Saanen and Toggenburg), and had strong genetic connectedness across both farm sites. Kids were removed from their mothers on the day of birth, reared on a milk replacement powder provided ad libitum via a machine, and weaned at 12 weeks of age. All weaned goats had constant access to fresh water and hay, and maiden females were fed a blended mix of cereals and legumes with molasses ad libitum. Females were put into mating groups containing between 30 and 50 individuals, once they reached a BW of $\sim 32 \mathrm{~kg}$. Mating groups were housed with a single male for 60 days, and all females were scanned for pregnancy between 30 and 60 days after removal of the male. After kidding, animals were milked three times per day in the first stage of lactation, which was reduced to twice a day when milk yields decreased. During first lactation, females were fed ad libitum for the first 150 days, at which point feed was restricted according to milk yield. Females that continued to yield high milk quantities at 10 months of lactation were retained in lactation, whereas lower yielding animals reentered mating groups after 10 months in lactation.

\section{Trait definitions}

Out of season kidding. Each day in the calendar year was assigned a week number, so that every date was assigned to the same week, regardless of year (e.g. 1 to 7 January=week 1).
First kidding (parturition) dates from 21270 goats were used to calculate the average number of kids born within each week, between the years 1987 and 2015. The 4 weeks of the year where the highest average number of births occurred, was defined as 'PKS', which roughly corresponded with the last week of February and the first 3 weeks of March in the calendar year. Each female in the final data set was assigned a value from 0 (animals that kidding in the spring - 'in season') to 24 (animals that kidded in the autumn - 'out of season'), depending on how many weeks either side of PKS she first kidded. The 'OOS' phenotype was therefore defined as the absolute week of kidding, relative to PKS. Only the first kidding was considered for analysis, as subsequent pregnancies will be influenced by the lactation length of each goat, as well as management practices, such as different lighting regimes.

Pseudopregnancy. Pseudopregnancy was recorded on a case-control basis. As very few records of PPG were available from nulliparous goats, PPG events that occurred during the first lactation were considered for analysis. All animals were scanned for pregnancy via trans-abdominal ultrasound, performed between 30 and 60 days after males were removed from mating groups. Cases of PPG were identified via the presence of uterine fluid in the absence of a foetus. In cases of PPG, prostaglandin was administered via intramuscular injection in order to induce discharge of the uterine fluid, and these females were returned to breeding groups. For the current analysis, cases of PPG were determined via records of prostaglandin administered between 1992 and 2013. Where records showed an animal had received prostaglandin within 200 days after her first kidding, within 150 days before her second kidding, or was administered a repeated dose of prostaglandin within a 60-day period, these records were removed from the data set. This is because these records were deemed too close to a previous or subsequent kidding date, or a previous dose of prostaglandin to be a true record of PPG. Females that did not receive prostaglandin during their first lactation between the years of 1992 and 2013, and were not excluded from the analysis during the quality control procedures described above, were selected as controls.

Age at first kidding. The age of goats at their first kidding (AFK) was measured in months. Records of AFK that fell outside 3 SD of the mean were excluded from the analysis.

Milk traits. Females with milk records for at least the first lactation, and for which entire productive lifetime records were available, were eligible for inclusion in the study. Animals with fewer than three milk records, that spent fewer than 90 days in milk (DIM), or that did not have milk records for the first lactation were excluded from the analysis.

Average DIM for lactation one was 443 days (SD 184.80). As goats typically have longer lactations than dairy cattle, and longer lactations are desirable to milk producers, a cumulative MY520 was considered for analysis. Milk yields for individuals with incomplete lactation records were 
projected up to 520 days, as per the Test Interval Method (International Committee for Animal Recording, 2003). When calculating MY520, yield records below 0.2 and above $12 \mathrm{~kg}$ were assumed to be anomalies and were excluded from the data set. Individuals with MY520 that fell beyond 3 SD of the mean were excluded from the final data set. Lifetime milk yield was the sum of the milk yield for each animal, across its entire productive life, whereas total DIMLife was calculated as the number of days between date of first kidding, and the final milk record.

\section{Characteristics of the data}

The final data set contained records from 9546 individuals, which were the progeny of 231 sires, and 7201 dams. A 14 generation pedigree containing 12617 animals was used for parameter estimations. Animals that met the criteria for each trait were included in the final data set, and each individual had a single record for each trait. Table 1 shows the descriptive statistics of the traits included in the analysis. Figure 1 shows the average number of kids born per week, across all years, centred around the peak kidding week, which was used to identify PKS. In total, $19 \%$ of females kidded during PKS $(n=1818), 63 \%$ of births took place between 1 and 12 weeks either side of PKS, and $18 \%$ of births occurred between 13 and 24 weeks outside of PKS.

Table 1 Characteristics of the traits out of season (OOS) kidding, age at first kidding (AFK), lifetime yield (MYLife), 520-day milk yield (MY520) and lifetime days in milk (DIMLife) included in the statistical analyses

\begin{tabular}{lrrrrr}
\hline \hline Traits & Mean & Median & \multicolumn{1}{c}{ SD } & Minimum & Maximum \\
\hline OOS $^{1}$ & 7.06 & 6 & 6.31 & 0.00 & 24.00 \\
AFK (months) & 15.51 & 15 & 3.01 & 9.00 & 25.00 \\
MY520 (kg) & 1429.16 & 1364 & 503.41 & 180.60 & 2889.30 \\
MYLife (kg) & 3056.31 & 2737 & 1967.56 & 43.98 & 9354.50 \\
DIMLife & 1164.54 & 1045 & 720.41 & 91.00 & 3452.00 \\
\hline \hline
\end{tabular}

${ }^{1}$ The absolute number of weeks of first kidding relative to peak kidding season, as defined by the 4 weeks of the year where the greatest number of kids are born.

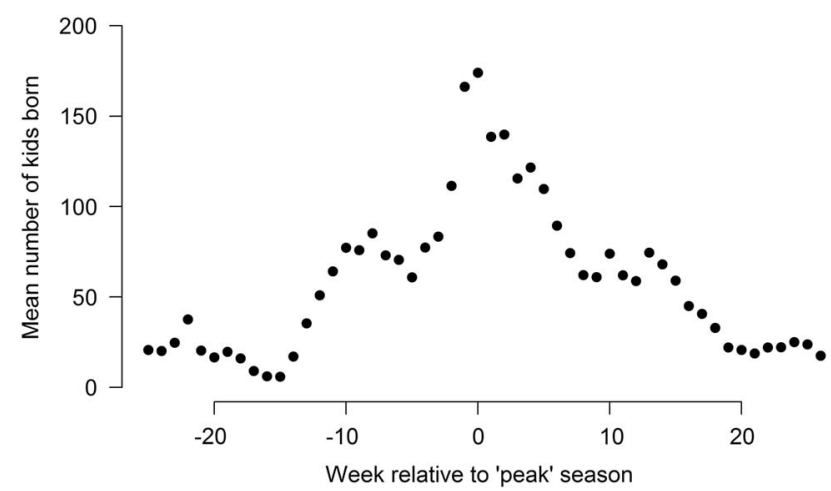

Figure 1 Average number of kids born per week between the years 1992 and 2013 in relation to the week with the highest average number of kids born (corresponded with late February/early March on the yearly calendar). Each day of the year was assigned to a week (e.g. 1 to 7 January = week 1 ).
Only $5 \%$ of females kidded for the first time between 21 and 24 weeks either side of PKS. The final data set contained 904 cases of PPG, and 8642 controls ( $10 \%$ incidence rate).

\section{Statistical analyses}

Genetic and environmental variance components were estimated via a series of univariate analyses using the following animal model:

$$
\mathbf{y}=\mathbf{X b}+\mathbf{Z a}+\mathbf{e}
$$

where $\mathbf{y}$ is the vector of observations recorded for a given trait, and the vectors $\mathbf{b}$, $\mathbf{a}$ and $\mathbf{e}$ represent the vectors of the fixed effects, additive genetic effects and residual error, respectively, and $\mathbf{X}$ and $\mathbf{Z}$ the incidence matrices relating records to fixed and additive genetic effects. Fixed effects fitted for each trait are reported in Table 2. Seasons were defined as summer (June to August), autumn (September to November), winter (December to February) and spring (March to May). Animals were assigned contemporary groups based on their kidding, or birth herd-year-season (see Table 2), and groups containing fewer than 50 animals were excluded from analysis. Fixed effects were retained in the model if they were found to be significant $(P<0.05)$, as determined by Wald $F$ statistics. Age at first kidding and dam age were not found to significantly affect PPG $(P=0.83$ and $P=0.08$, respectively) and so were excluded from the final model. Unfortunately, there was no available information on litter size born, BW at mating, mating group composition or the number/timing of mating attempts for individual animals, therefore it was not possible to consider these effects in the model.

Pseudopregnancy was measured as a binary trait, therefore a threshold animal model (Gianola and Foulley, 1983) was applied using a logit link function (Gilmour et al., 2009) with $\eta=\log \left(\frac{m}{1-m}\right)$, where $m$ is the mean on the observed scale and $\boldsymbol{\eta}$ the vector of linear predictors of liability of PPG

Table 2 Fixed effects included in the univariate analyses for the reproduction traits out of season (OOS) breeding, pseudopregnancy (PPG) and age at first kidding (AFK), and the production traits 520-day milk yield (MY520), lifetime yield (MYLife) and lifetime days in milk (DIMLife)

\begin{tabular}{|c|c|c|c|}
\hline Traits & Fixed effect & $\mathrm{DF}$ & $P$ value \\
\hline \multirow[t]{2}{*}{$00 \mathrm{~S}$} & Herd-year-season of birth & 73 & $<0.001$ \\
\hline & AFK (months) & 16 & $<0.001$ \\
\hline \multirow[t]{2}{*}{ PPG } & Herd-year-season of kidding & 63 & $<0.001$ \\
\hline & Year-season of birth & 58 & 0.030 \\
\hline AFK & Herd-year-season of birth & 73 & $<0.001$ \\
\hline \multirow[t]{2}{*}{ MY520 ( $1^{\text {st }}$ lactation $)$} & Herd-year-season of kidding & 63 & $<0.001$ \\
\hline & Lactation length (days)* & 1 & $<0.001$ \\
\hline \multirow[t]{3}{*}{ MYLife (kg) } & Herd-year-season of birth & 73 & $<0.001$ \\
\hline & Number of lactations ( 1 to 11 )* & 1 & $<0.001$ \\
\hline & DIMLife* & 1 & $<0.001$ \\
\hline \multirow{3}{*}{ DIMLife } & Herd-year-season of birth & 73 & $<0.001$ \\
\hline & MYLife $(\mathrm{kg})^{*}$ & 1 & $<0.001$ \\
\hline & Number of lactations ( 1 to 11 )* & 1 & $<0.001$ \\
\hline
\end{tabular}

*Fitted as linear covariate. 
on the underlying scale estimated as $\boldsymbol{\eta}=\mathbf{X} \boldsymbol{\varphi}$, with $\mathbf{X}$ as an incidence matrix and $\boldsymbol{\varphi}$ a vector of regression variables. As with binary data the threshold and the residual variance are not identifiable, these parameters were set to arbitrary values $\left(\tau=0\right.$ and $\left.\sigma^{2}=\pi^{2} / 3 \sim 3.29\right)$. The resulting model can be summarized as:

$$
\eta=\mathrm{Xb}+\mathrm{Za}+\mathrm{e}
$$

where $\boldsymbol{\eta}$ is the liability of having PPG, $\mathbf{b}$ a vector of fixed effects containing $\mu$ - liability of PPG and fixed effect as detailed in Table 2, $\mathbf{a}$ and $\mathbf{e}$ are as defined above, $\mathbf{X}$ and $\mathbf{Z}$ are incidence matrices that link fixed and random animal effects to the liability of PPG.

The variance-covariance structure of the fitted models was as follows:

$$
\operatorname{Var}\left[\begin{array}{l}
a \\
\mathbf{e}
\end{array}\right]=\left[\begin{array}{cc}
\mathbf{A} \sigma_{g}^{2} & 0 \\
0 & \mathbf{I} \sigma_{e}^{2}
\end{array}\right]
$$

where $\mathbf{A}$ and $\mathbf{I}$ are the additive genetic relationship matrix and identity matrix, respectively. A series of bivariate analyses were performed between reproduction and milk traits. Where fixed effects included in the univariate model were confounded with the second trait of interest in the bivariate model, that fixed effect was removed. For example, AFK was included as a fixed effect in the univariate model for PPG, but was removed when calculating correlations between these two traits. Fixed and random effects fitted for each trait were based on the univariate analyses as presented in Table 1 . The following covariance structure was used for the bivariate analyses:

$$
\operatorname{Var}\left[\begin{array}{l}
a_{1} \\
a_{2} \\
e_{1} \\
e_{2}
\end{array}\right]=\left[\begin{array}{cccc}
\mathbf{A} \sigma_{g 1}^{2} & \mathbf{A} \sigma_{g 12} & 0 & 0 \\
& \mathbf{A} \sigma_{g 2}^{2} & 0 & 0 \\
& & \mathbf{I} \sigma_{e 1}^{2} & \mathbf{I} \sigma_{e 12} \\
\text { symm } & & & \mathbf{I} \sigma_{e 2}^{2}
\end{array}\right]
$$

where indices 1 and 2 indicate the 2 traits. The first trait was one of the reproduction traits, and the second trait was one of the milk traits, $\mathbf{A}$ is the additive genetic relationship matrix, I are identity matrices, and $\sigma_{g}^{2}$ and $\sigma^{2}{ }_{e}$ the genetic and residual variances, respectively. All genetic analyses were performed using ASReml (Gilmour et al., 2009).

\section{Results}

\section{Parameters}

Heritabilities and variance components for each trait are presented in Table 3. The heritabilities for traits related to reproduction were of a low magnitude (0.08 to 0.11$)$. Heritabilities for the production traits MY520, MYLife and DIMLife ranged from 0.12 to 0.35 .

\section{Correlations}

Out of season kidding. All correlations are presented in Table 4. Genetic correlations between production traits and OOS were of a low magnitude and associated with a high
Table 3 Heritabilities $\left(\mathrm{h}^{2}\right)$, genetic $\left(\sigma^{2}{ }_{\mathrm{a}}\right)$ and phenotypic variances $\left(\sigma_{p}^{2}\right)$ for the reproduction traits out of season (OOS) breeding, pseudopregnancy (PPG) and age at first kidding (AFK), and the production traits 520-day milk yield (MY520), lifetime yield (MYLife) and lifetime days in milk (DIMLife)

\begin{tabular}{lcrr}
\hline \hline Traits & $h^{2}$ & \multicolumn{1}{c}{$\sigma^{2}{ }_{a}$} & \multicolumn{1}{c}{$\sigma^{2}{ }_{p}$} \\
\hline OOS kidding $^{1}$ & $0.11(0.02)$ & 2.32 & 20.92 \\
PPG & $0.11(0.02)$ & 0.39 & 3.68 \\
AFK (months) & $0.08(0.02)$ & 0.40 & 5.29 \\
MY520 (kg) & $0.35(0.03)$ & 69347.00 & 195640.00 \\
MYLife (kg) & $0.20(0.02)$ & 143868.00 & 731770.00 \\
DIMLife & $0.12(0.02)$ & 5547.08 & 45595.00 \\
\hline \hline
\end{tabular}

${ }^{1}$ The absolute number of weeks of first kidding relative to peak kidding season, as defined by the 4 weeks of the year where the greatest number of kids are born.

${ }^{2}$ First lactation.

standard error. Phenotypic correlations between these traits were close to 0 .

Pseudopregnancy. At the phenotypic level, low to very low positive correlations were found between PPG, and MY520 $(0.09 \pm 0.02)$, DIMLife $(0.29 \pm 0.01)$, AFK $(0.06 \pm 0.02)$ and OOS kidding $(0.06 \pm 0.02)$. In contrast, very low negative phenotypic correlations were estimated between PPG and MYLife $(-0.08 \pm 0.02)$. At the genetic level, PPG was found to be moderately positively correlated with DIMLife $(0.58 \pm 0.11)$, and with 00 S kidding $(0.36 \pm 0.15)$. As higher values for 005 related to kidding dates further away from PKS, this means that animals that breed $00 \mathrm{~S}$ were more likely to experience PPG. All other correlations were found to be very low and associated with a large standard error (Table 4).

Age at first kidding. The phenotypic correlation between AFK and MY520 was very weak and positive $(0.07 \pm 0.01)$. Conversely, the genetic correlation was low and negative $(-0.22 \pm 0.10)$, meaning that kidding at a young age was genetically associated with higher first lactation milk yields. All other correlations between production traits and AFK were either close to 0 , or associated with a high standard error (Table 3). Out of season breeding and AFK were moderately, negatively correlated on the phenotypic level, but not genetic level, meaning that does that kidded at a younger age were more likely to do so outside of PKS.

\section{Discussion}

\section{Heritabilities of production traits}

Heritability for MY520 was within the expected range for this population, based on a random regression analysis on a larger sample of the present population (Mucha et al., 2014) and in line with estimates for dairy ewes and goats (El Saied et al., 2005; Rupp et al., 2011; García-Peniche et al., 2012; Castañeda-Bustos et al., 2014). Heritability for DIMLife was similar to that estimated by Torrero $(2010 ; 0.13)$, and lower 
Table 4 Genetic $\left(r_{G}\right)$, residual $\left(r_{R}\right)$ and phenotypic $\left(r_{P}\right)$ correlations (SE) between out of season (OOS) breeding, pseudopregnancy (PPG), age at first kidding (AFK), with the production traits lifetime yield (MYLife), 520-day milk yield (MY520) and lifetime days in milk (DIMLife)

\begin{tabular}{|c|c|c|c|c|c|c|}
\hline \multirow[b]{2}{*}{ Traits } & \multicolumn{2}{|c|}{$\operatorname{OOS}^{1}$} & \multicolumn{2}{|c|}{ AFK } & \multicolumn{2}{|c|}{ PPG } \\
\hline & $r_{G}$ & $r_{P}$ & $r_{G}$ & $r_{P}$ & $r_{G}$ & $r_{P}$ \\
\hline MY520 (kg) & $-0.15(0.09)$ & $0.01(0.01)$ & $-0.22(0.10)$ & $0.07(0.01)$ & $-0.03(0.11)$ & $0.09(0.02)$ \\
\hline MYLife (kg) & $-0.17(0.10)$ & $-0.02(0.01)$ & $-0.04(0.11)$ & $0.01(0.01)$ & $-0.09(0.13)$ & $-0.08(0.02)$ \\
\hline DIMLife & $0.14(0.11)$ & $0.01(0.01)$ & $0.15(0.13)$ & $-0.02(0.01)$ & $0.58(0.11)$ & $0.29(0.01)$ \\
\hline PPG & $0.36(0.15)$ & $0.06(0.02)$ & $-0.26(0.14)$ & $0.06(0.02)$ & - & - \\
\hline AFK & $0.02(0.16)$ & $-0.32(0.01)$ & - & - & - & - \\
\hline
\end{tabular}

${ }^{1}$ The absolute number of weeks of first kidding relative to peak kidding season, as defined by the 4 weeks of the year where the greatest number of kids are born.

than the heritability of 0.22 estimated for a similar trait estimated by Castañeda-Bustos et al. (2014).

\section{Out of season breeding}

The ability to breed at any time of year would help alleviate milk supply problems associated with the natural seasonal fluctuation in birth rates. It would also allow for faster genetic improvement via accelerated breeding. Variation in breeding season between and within breeds has been shown to exist, even when managed under equivalent conditions, and studies in sheep have shown a heritable component to seasonality (Hanocq et al., 1999; Chemineau et al., 2010).

Defining and measuring the capability to breed OOS is challenging, as recording the start and end of oestrus for individual animals in a large, naturally bred milking herd is impractical. One of the objectives of the present study, was to develop a method of phenotyping OOS breeding ability using routinely collected kidding dates. There were two main factors that presented a challenge when analysing the data. First, goats reach sexual maturity and are bred at around 10 months of age (Greyling, 1990), therefore the birth date of any given goat influenced whether she was given the opportunity to breed OOS for the first time. To adjust for this effect, the animal's own birth herd-year-season was included as a fixed effect, to account for any variance explained by the time in the year an animal was born. Second, an older goat may have had several opportunities to mate before her first kidding, therefore her kidding date may not reflect her true seasonal breeding ability. In the absence of breeding records, the age of the animal at kidding was also fitted as a fixed effect in the model. Both of these factors were found to significantly affect this trait, however, birth herd-year-season was found to account for a higher proportion of the variance, compared with AFK (results not presented).

In this study, 00S kidding was defined as the absolute number of weeks of kidding, relative to PKS, and no distinction was made between animals that kidded before or after PKS. The disadvantage of this methodology is that a breeder would be unable to discern between animals that kidded earlier or later than PKS, should one of these traits be considered of greater value. As illustrated in Figure 1, kidding seasonality is cyclical in nature, with birth rates steadily reducing after PKS, and rising as PKS approaches. Distinguishing between goats that kidded before or after PKS therefore creates difficulties when defining a linear phenotype. For example, in a linear model, two animals assigned phenotypes of -24 and +24 weeks relative to PKS would be treated as separate traits, when in reality both phenotypes represent autumn kidding. Defining the phenotype in this way would have the effect of reducing the proportion of variance attributed to genetic effects in the analysis. This would also make genetic correlations between OOS and other traits difficult to interpret, as very low and high values would be biologically similar, but would have very different breeding values. A similar problem would occur if 005 was simply defined as the week of kidding in the calendar year, without centring birth dates around a PKS.

Out of season breeding, as defined in the current study, was found to have a low estimated heritability (0.11). The genetic basis of $00 \mathrm{~S}$ kidding has not previously been explored in depth in dairy goats, however, several studies in sheep have found a genetic component to traits related to seasonality (Quirke et al., 1986; Chemineau et al., 2010), and selection for fall lambing has been achieved (Vincent et al., 2000). Smith et al. (1992) estimated a heritability of 0.23 for seasonal breeding ability, based on a binary trait of successful/unsuccessful breeding in the late spring/summer months. It was not possible to directly investigate out of season breeding success in this study, as detailed mating opportunity records were unavailable.

Genetic correlations between 00S kidding and MY520, MYLife or DIMLife were of a very low magnitude. This suggests that selection for this trait would not adversely affect milk yield, however, milk yield is only one of many traits of importance to breeders. Seasonal breeding is a polygenic trait, involving many physiological processes (Chemineau et al., 2010), therefore it is likely to be genetically related to other traits, particularly those relating to fertility.

Age at first kidding was accounted for in the univariate model for OOS, and moderate negative phenotypic correlations between these two traits suggested that animals that kid OOS for the first time are more likely to kid at a younger age, although this was not observed on a genetic level. As oestrus is not recorded in this population, age and BW are 
used to gauge sexual maturity. Goats that attain a suitable BW outside of the breeding season may need to wait longer until first breeding, which would explain the negative phenotypic correlation observed between 0OS kidding and AFK. This limits the interpretation as it is not known whether an older kidding age was due to aseasonal infertility (i.e. she tried and failed to breed 00S), or if she simply was not given the opportunity to breed.

\section{Pseudopregnancy}

The present paper is the only known study that has estimated the heritability for PPG using classic quantitative genetic methodology. Hesselink and Elving (1996) observed incidence rates of between $5 \%$ and $21 \%$ in a herd of dairy goats recorded over the course of 4 years. They found that PPG occurred in $38 \%$ of daughters of goats that had been known to suffer a PPG, compared with just $9 \%$ of daughters from unaffected dams, suggesting a genetic component to the trait.

There is a strong positive phenotypic correlation between MYLife and DIMLife $(0.88 \pm 0.00)$, therefore we would expect correlations between these traits and PPG to be similar. To disentangle the effects due to collinearity between these traits, milk yield was adjusted for lactation length (and vice versa) in the models. Pseudopregnancy within the first lactation was phenotypically associated with higher milk yield in that lactation. As lactation length was included as a covariate, this association was not simply due to longer lactation lengths arising from PPG. This association was not reflected at the genetic level, suggesting that the relationship is likely to be due to environmental factors, rather than the inherent biology of the animal. Conversely, a low, negative, phenotypic correlation was found between PPG and MYLife, suggesting that females that experience PPG in the first lactation will produce slightly less milk overall, even when they remain in the herd for equivalent lengths of time. This relationship may be reflected at the genetic level, although high standard errors limit that interpretation. After adjusting for milk yield, there were moderate, positive genetic and phenotypic correlations between PPG and DIMLife, suggesting that animals that experience PPG in their first lactation produce equivalent milk yields over a longer period of time. These results suggest an unfavourable relationship between PPG and lifetime production.

To the author's knowledge, there are no other studies that have attempted to quantify the genetic correlations between the incidence of PPG with milk production traits. At present, it is unclear what the economic costs of PPG are, and whether this phenomenon is of major concern to dairy goat farmers, due to the fact that it extends lactation, without producing a potentially unwanted kid. Souza et al. (2013) suggested that PPG may be associated with lower fertility, which may be problematic in the long run, if present at the genetic level. As discussed above, the results of this study suggest that although PPG extends lactation time, this is not associated with higher milk yield, therefore there does not appear to be a reason for a producer to desire higher levels of PPG in a herd. In cases where a kid is wanted, for example, in meat production or when aiming for genetic improvement, PPG may be a specific cause for concern, as the extended period of time between kidding will hinder progress. As PPG cannot easily be verified within 30 days after mating/insemination this can add a significant delay in creating replacement animals, or commencing new lactations, across a herd. Further work would be required to quantify the cost of PPG to producers.

There is some evidence that PPG may be more prevalent outside of the normal breeding season (Duquesnel et al., 1992), therefore selection for aseasonal breeding may exacerbate this problem. In this population, the correlations suggest that females that gave birth for the first time far outside of PKS were more likely to experience PPG during their next breeding attempt. As cases of PPG were determined via ultrasound performed between 30 and 60 days after mating, it was impossible to tell with any degree of accuracy exactly when mating that led to a PPG occurred. This in turn meant it was not possible to correlate PPG with season of breeding. Information from pregnancy scanning records suggests that the highest number of PPG were detected in June ( $25 \%$ of all cases), which corresponds to a rough mating opportunity window of between 1 and 3 months previously, however, this figure will reflect the fact that more matings occur in the autumn and winter months (the natural breeding season). The lowest rates of PPG were detected in September ( $<1 \%$ of cases), which would correspond to spring and summer breeding, however, fewer animals are mated in summer due to the low success rate. As OOS kidding as defined here has not been correlated with direct measures of mating opportunity, it is not certain whether these results accurately reflect a true genetic association between PPG and aseasonal breeding.

\section{Age at first kidding}

The genetic component of AFK has been well studied in goats. In the current study the heritability for AFK was low $(0.08)$, but within a range and with sufficient genetic variance to suggest this trait would respond to selection. The heritability for this trait was lower than those estimated in the United States $(0.23 \pm 0.02$ - García-Peniche et al., 2012; $0.16 \pm 0.01$ - Castañeda-Bustos et al., 2014), Polish $(0.13 \pm 0.04$ - Bagnicka et al., 2007), Ethiopian $(0.25 \pm 0.19$ - Kebede et al., 2012) and Mexican dairy goat populations $(0.31 \pm 0.09$ - Torres-Vázquez et al., 2009). With the exception of Bagnicka et al. (2007), for which it was higher, genetic variance was found to be between $80 \%$ and $89 \%$ lower in this population, compared with the studies cited above, despite being based on a larger number of records in most cases. The management of the herd in the present study was such that animals were bred upon reaching a mature weight of $\sim 32 \mathrm{~kg}$. This practice may explain the low heritability and genetic variance in this population.

The very low, positive, phenotypic correlation between AFK and MY520 suggests that goats that first kid at an older age produce slightly more milk within the first lactation. This result was also found in dairy cattle (Pirlo et al., 2000). 
The association may be due to the fact that older goats are closer to their full, mature weight, and are therefore able to allocate more resources to lactation (Pérez-Razo et al., 2004). The contrasting low, negative genetic correlation between AFK and MY520 suggests that younger AFK may be genetically associated with higher MY520 within the first lactation, although this correlation was associated with a high standard error. Age at first kidding did not correlate with DIMLife or MYLife, therefore AFK does not seem to be associated with a longer or higher yielding productive life. Our results are in agreement with Torres-Vázquez et al. (2009) who calculated genetic correlations for AFK with 305day milk, fat and protein yield of $-0.18,-0.09$ and -0.17 , respectively, although they were associated with extremely large standard errors. Kennedy et al. (1982) estimated a very low genetic correlation of -0.05 between AFK and milk yield in dairy goats. On a phenotypic level, Pérez-Razo et al. (2004) found that increased AFK was simultaneously associated with higher stayability - defined as the proportion of animals that remain productive until a fixed end point (Pellerin and Browning, 2012) - and lower number of lactations. Castañeda-Bustos et al. (2014) found a low, negative genetic $(-0.03 \pm 0.06)$ correlation between AFK and productive life at 72 months (defined as the total number of days in production recorded up to 72 months of age), which is the opposite direction to that estimated in the present study, however, given the high error of estimation in both studies it is difficult to predict the true association between these traits.

Reducing AFK is advantageous as it reduces the cost of managing unproductive members of the herd. In addition, reducing AFK also reduces generation interval, which speeds up genetic improvement, and may be associated with improved performance. Conversely, there may be a trade-off between rearing cost savings, and potential unfavourable associations between AFK and milk yield and longevity. Several studies in cattle have suggested that optimal profitability may not be achieved via calving at the youngest possible age, and that an intermediate age may be preferable (Gill and Allaire 1976; Pirlo et al., 2000; Nilforooshan and Edriss 2004).

\section{Conclusion}

Our study shows that fertility traits in dairy goats generally have a low heritability, yet are well within published ranges in other studies for similar traits, suggesting that these traits would respond to selection pressure. The results presented here suggest that selection for younger AFK would be possible without adversely affecting milk output. Accurately measuring seasonal breeding ability is challenging and time consuming. Out of season kidding as defined here offers an easily recorded proxy measure of aseasonal breeding ability. The correlation of OOS kidding with other traits suggest that it may be possible to select for aseasonal breeding, using routinely collected kidding dates, without adversely affecting production. However, our results also suggest that animals with greater ability to breed OOS are also more likely to experience PPG. The present study is the first to estimate heritability of PPG. Our results show that PPG is associated with longer productive lifespan, however, this is independent of milk yield. The reproductive traits discussed in this study are likely to have high economic value, but have low heritability, few records per animal (either due to costly recording procedures, or infrequent expression of the trait), and are expressed later in life. Genetic gain via conventional breeding programmes will therefore be slow, due to low accuracy and high generation interval. As such, traits such as these are particularly suited to genomic selection (Shumbusho et al., 2013), and future work should investigate genomic breeding values for these traits, alongside other traits of economic importance.

\section{Acknowledgements}

This work was supported by the Biotechnology and Biological Sciences Research Council (grant number BB/M027570/1) and Innovate UK. The authors gratefully acknowledge cooperation with staff from Yorkshire Dairy Goats.

\section{References}

Bagnicka E, Wallin E, Łukaszewicz M and Ådnøy T 2007. Heritability for reproduction traits in Polish and Norwegian populations of dairy goat. Small Ruminant Research 68, 256-262.

Castañeda-Bustos VJ, Montaldo HH, Torres-Hernández G, Pérez-Elizalde $\mathrm{S}$, Valencia-Posadas M, Hernández-Mendo 0 and Shepard L 2014. Estimation of genetic parameters for productive life, reproduction, and milk-production traits in US dairy goats. Journal of Dairy Science 97, 2462-2473.

Chemineau P, Bodin L, Migaud M, Thiéry JC and Malpaux B 2010. Neuroendocrine and genetic control of seasonal reproduction in sheep and goats. Reproduction in Domestic Animals 45, 42-49.

Duquesnel R, Parisot D, Pirot G, Mialot JP, Saboureau L, Etienne P, Delaval J, Guéraud JM, Prengere $E$, de Montigny $G$, Guerrault $P$, Perrin $G$, Humblot $P$, de Fontaubert $Y$ and Chemineau $P$ 1992. Pseudopregnancy in the goat. Annales de Zootechnie 41, 407-415.

El-Saied UM, De La Fuente LF, Carriedo JA and San Primitivo F 2005. Genetic and Phenotypic Parameter Estimates of Total and Partial Lifetime Traits for Dairy Ewes. Journal of Dairy Science 88, 3265-3272.

García-Peniche TB, Montaldo HH, Valencia-Posadas M, Wiggans GR, Hubbard SM, Torres-Vázquez JA and Shepard L 2012. Breed differences over time and heritability estimates for production and reproduction traits of dairy goats in the United States. Journal of Dairy Science 95, 2707-2717.

Gianola D and Foulley J 1983. Sire evaluation for ordered categorical data with a threshold model. Genetics, Selection, Evolution 15, 201-224.

Gill GS and Allaire FR 1976. Relationship of age at first calving, days open, days dry, and herdlife to a profit function for dairy cattle. Journal of Dairy Science 59, 1131-1139.

Gilmour AR, Gogel BJ, Cullis BR and Thompson R 2009. ASReml user guide release 3.0. VSN International Ltd., Hemel Hempstead, UK.

Greyling PC 1990. Puberty and the induction of puberty in female Boer goat kids. South African Journal of Animal Science 20, 193-200.

Hanocq E, Bodin L, Thimonier J, Teyssier J, Malpaux B and Chemineau P 1999. Genetic parameters of spontaneous spring ovulatory activity in Mérinos d'Arles sheep. Genetique Selection Evolution 31, 77-90.

Hesselink JW 1993. Hydrometra in dairy goats: reproductive performance after treatment with prostaglandins. The Veterinary Record 133, 186-187.

Hesselink JW and Elving L 1996. Pedigree analysis in a herd of dairy goats with respect to the incidence of hydrometra. The Veterinary Quarterly 18, 24-25.

International Committee for Animal Recording 2003. Guidelines approved by the General Assembly held in Interlaken, Switzerland. 30 May 2002, Roma. 


\section{Desire, Mucha, Coffey, Mrode, Broadbent and Conington}

Kebede T, Haile A, Dadi H and Alemu T 2012. Genetic and phenotypic parameter estimates for reproduction traits in indigenous Arsi-Bale goats. Tropical Animal Health and Production 44, 1007-1015.

Kennedy BW, Finley CM and Bradford GE 1982. Phenotypic and genetic relationships between reproduction and milk production in dairy goats. Journal of Dairy Science 65, 2373-2383.

Leboeuf B, Renaud G, De Fontaubert Y, Broqua B and Chemineau P 1994. Echographie et pseudogestation chez la diagnosis with real time ultrasound and treatment with prosta- chevre. In Proceedings of the 7th International Meeting on Animal Reproduction, pp. 251-255.

Mucha S, Mrode R, Coffey M and Conington J 2014. Estimation of genetic parameters for milk yield across lactations in mixed-breed dairy goats. Journal of Dairy Science 97, 2455-2461.

Nilforooshan MA and Edriss MA 2004. Effect of age at first calving on some productive and longevity traits in Iranian Holsteins of the Isfahan province. Journal of Dairy Science 87, 2130-2135.

Pellerin AN and Browning R 2012. Comparison of Boer, Kiko, and Spanish meat goat does for stayability and cumulative reproductive output in the humid subtropical southeastern United States. BMC Veterinary Research 8, 136.

Pérez-Razo M, Sánchez F, Torres-Hernándezc G, Becerril-Pérez C, GallegosSánchez J, González-Cosío F and Meza-Herrera C 2004. Risk factors associated with dairy goats stayability. Livestock Production Science 89, 139-146.

Pirlo G, Miglior F and Speroni M 2000. Effect of age at first calving on production traits and on difference between milk yield returns and rearing costs in Italian Holsteins. Journal of Dairy Science 83, 603-608.

Quirke JF, Hanrahan JP, Loughnane W and Triggs R 1986. Components of the breeding and non-breeding seasons in sheep: breed effects and repeatability. Irish Journal of Agricultural Research 25, 167-172.
Rauw W, Kanis E, Noordhuizen-Stassen E and Grommers F 1998. Undesirable side effects of selection for high production efficiency in farm animals: a review. Livestock Production Science 56, 15-33.

Rupp R, Clément V, Piacere A, Robert-Granié C and Manfredi E 2011. Genetic parameters for milk somatic cell score and relationship with production and udder type traits in dairy Alpine and Saanen primiparous goats. Journal of Dairy Science 94, 3629-3634.

Shumbusho F, Raoul J, Astruc J, Palhiere I and Elsen JM 2013. Potential benefits of genomic selection on genetic gain of small ruminant breeding programs. Journal of Animal Science 91, 3644-3657.

Smith JF, Johnson DL and Reid TC 1992. Genetic parameters and performance of flocks selected for advanced lambing date. Proceedings of the New Zealand Society of Animal Production 52, 129-131.

Souza JMG, Maia ALRS, Brandão FZ, Vilela CG, Oba E, Bruschi JH and Fonseca JF 2013. Hormonal treatment of dairy goats affected by hydrometra associated or not with ovarian follicular cyst. Small Ruminant Research 111, 104-109.

Torrero GY 2010. Estimación de covarianzas para características de longevidad y producción en cabras. MSc Thesis, University of Guanajuato, Irapuato, Guanajuato, Mexico.

Torres-Vázquez JA, Valencia-Posadas $\mathrm{M}$, Castillo-Juárez $\mathrm{H}$ and Montaldo $\mathrm{HH}$ 2009. Genetic and phenotypic parameters of milk yield, milk composition and age at first kidding in Saanen goats from Mexico. Livestock Science 126, 147-153.

Vincent JN, McQuown EC and Notter DR 2000. Duration of the seasonal anestrus in sheep selected for fertility in a fall-lambing system. Journal of Animal Science 78, 1149-1154. 